\title{
On the asymptotic spatial behaviour of the solutions of the nerve system
}

\author{
M.C. Leseduarte and R. Quintanilla \\ Matemática Aplicada 2 Universidad Politècnica de Catalunya \\ Colom, 11. Terrassa. Barcelona. Spain
}

Keywords: Nerve equation, spatial decay, estimates, spatial nonexistence, FitzHugh Nagumo.

\begin{abstract}
In this paper we investigate the asymptotic spatial behavior of the solutions for several models for the nerve fibers. First, our analysis deals with the coupling of two parabolic equations. We prove that, under suitable assumptions on the coefficients and the nonlinear function, the decay is similar to the one corresponding to the heat equation. A limit case of this system corresponds to the coupling of a parabolic equation with an ordinary differential equation. In this situation, we see that for suitable boundary conditions the solution ceases to exist for a finite value of the spatial variable. Next two sections correspond to the coupling of a hyperbolic/parabolic and hyperbolic/ordinary differential problems. For the first one we obtain that the decay is like an exponential of a second degree polynomial in the spatial variable. In the second one, we prove a similar behaviour to the one corresponding to the wave equation. In these two sections we use in a relevant way an exponentially weighted Poincaré inequality which has been revealed very useful in several thermal and mechanical problems. This kind of results have relevance to understand the propagation of perturbations for nerve models.
\end{abstract}

\section{Introduction}

Hodgkin and Huxley [11] proposed a mathematical model to describe the propagation of electrical impulses in nerve fibers. This is a system of an ordinary differential equation coupled with a parabolic equation. A simpler model which describes the same qualitative behaviour is constituted by the equations proposed by FitzHugh [5] and Nagumo, Arimoto and Yoshizawa [19]. This system of equations is usually called FitzHugh-Nagumo equations and takes the form

$$
\begin{gathered}
v_{, t}=v_{, i i}+f(v)-u, \\
u_{, t}=\sigma v-\gamma u,
\end{gathered}
$$

where $\sigma$ and $\gamma$ are constants. In this system $f(v)$ is a nonlinear function satisfying (2.5). A paradigm for these functions are those of the form $f(v)=-a|v|^{n} v+b|v|^{\frac{n}{2}} v+\mu_{1} v$, where $n, a$ and $\mu_{1}$ are positive constants ${ }^{1}$. The system is defined in a cylinder $R$ along a period of time.

\footnotetext{
${ }^{1}$ When $n=2$, we get the nonlinear term usually appearing in FitzHugh-Nagumo equations.
} 
The spatial variable $\boldsymbol{x}$ belongs to the cylinder $R$ and the time $t \in(0, \infty)$. The variable $v(\boldsymbol{x}, t)$ describes the membrane voltage and $u(\boldsymbol{x}, t)$ is an auxiliar function (see [16]). Some people have considered the generalization of the system of equations and now it is usual to study the extension of the equations of FitzHugh-Nagumo constitute by the equation (1.1) and the diffusion equation

$$
u_{, t}=\delta u_{, i i}+\sigma v-\gamma u
$$

where $\delta$ is a positive constant.

In 1967, Lieberstein [16] suggested to change the diffusion equation in the model of Hodgkin and Huxley. He used some equations which are more general than the ones used by FitzHugh and Nagumo. These arguments suggested to consider what it is known as the hyperbolic version of the FitzHugh-Nagumo system of equations. In this case, it is usual to change the diffusion equation (1.1) by the hyperbolic one

$$
\epsilon v_{, t t}+\left(1-\epsilon f^{\prime}(v)\right) v_{, t}=v_{, i i}+f(v)-u,
$$

where $\epsilon$ is a positive constant and $f(v)$ is a function that satisfies condition (4.2). It is worth noting that if in this system we take $\epsilon=0$, we obtain the usual system of the FitzHugh-Nagumo equations. These systems have deserved much attention in recent years (see $[2,10,14,17,23$, 26,27 ], among others) from a mathematical point of view. In this paper we want to analyse an aspect which has not been considered yet in the literature. We want to study the spatial behavior of the solutions and in particular its asymptotic decay.

The systems of equations we propose describe the propagation of the excitation along the nerve axon. The spatial decay estimates for these problems will play a relevant role to understand the influence of the stimuli far away from the place where it is applied. Results on asymptotic spatial decay of solutions have been obtained mainly in thermomechanical problems $[4,6,7,12,13,20,21,24,25]$. The motivation is given in the context of the Saint-Venant principle. We believe that this kind of studies are also a valuable objective in the context of the propagation of the excitation in nerve models. Such studies give information concerning how the signals propagate along the nerve and how the disturbances are damped far away where they have been produced.

In this paper we denote by $R$ the cylinder of the form $(0, \infty) \times D$, where $D$ is a two dimensional bounded domain such that the boundary $\partial D$ is smooth enough to apply the divergence theorem. By $D(z)$ we denote the cross section of the points in $R$ such that $x_{1}=z$, by $R(z)$ we mean the points of the cylinder such that $x_{1}>z$.

In the next sections we will use the following fact (see the Appendix of [15] for a proof):

Exponentially weighted Poincaré inequality. Assume that $g(s)$ is a differentiable function in $[0, t]$ such that $g(0)=0$. Then the following inequality

$$
\int_{0}^{t} \exp (-2 \omega s) g^{2}(s) d s \leq \frac{4 t^{2}}{\pi^{2}+4 t^{2} \omega^{2}} \int_{0}^{t} \exp (-2 \omega s)(\dot{g}(s))^{2} d s
$$

holds, for every $\omega>0$. We note that $\varphi(t)=\frac{4 t^{2}}{\pi^{2}+4 t^{2} \omega^{2}}$ is a growing function and so

$$
\int_{0}^{t} \exp (-2 \omega s) g^{2}(s) d s \leq \frac{4 t_{0}^{2}}{\pi^{2}+4 t_{0}^{2} \omega^{2}} \int_{0}^{t} \exp (-2 \omega s)(\dot{g}(s))^{2} d s, 0<t \leq t_{0} .
$$


The content of this paper is as follows. In Section 2 we consider a problem for the system determined by (1.1) and (1.3). As it is the coupling of two diffusion equations, we will prove that an estimate similar to the one proposed for the Fourier heat equation can be applied in this context. To overcome the difficulty proposed by the coupling and the nonlinear terms, we use a weighted measure. We will see that the decay can be controlled by an exponential of the square of the distance to the cylinder base (see (2.35)). In Section 3 we consider the system determined by (1.1) and (1.2). A similar argument to the one considered in Section 2 can be obtained, but we here want to emphasize in a spatial nonexistence theorem when the nonlinear term satisfies a certain condition (see (3.2)) which is usually related with the conditions proposed in the literature. Section 4 is devoted to the coupled system (1.4) and (1.3). That is the coupling of an hyperbolic equation with a parabolic equation. We will see how the solutions decay as the exponential of a second order polynomial of the distance to the base. It is worth noting that this fact is very usual in the context of mixtures of parabolic and hyperbolic equations. However, the nonlinear part of this system proposes several difficulties to obtain the estimates. In our case we need to use the exponentially weighted Poincaré inequality and, in particular, the proposed bound (1.6) to overcome the difficulty. This inequality has been revealed recently very useful to solve several difficulties in the study of the spatial decay estimates (see [15]). In Section 5 we consider the coupling of equations (1.4) and (1.2). We prove that the usual arguments for the hyperbolic problems can be applied to this situation. A domain of influence result is a corollary. We also need to use the upper bound for the exponentially weighted Poincaré inequality proposed at (1.6) and in particular a domain of influence theorem is obtained. In the last section we summarize the main results of the paper. Finally, we add an Appendix where we show some properties of the function $f(v)$ appearing in the systems of equations for the different sections.

In this paper we are not concerning about existence on solutions. The existence as well as the regularity of several type for the solutions are assumed.

\section{Parabolic problem}

In this section we obtain bounds for the spatial decay of the solutions of the problem determined by the system (1.1), (1.3), the initial conditions

$$
v(\boldsymbol{x}, 0)=u(\boldsymbol{x}, 0)=0, \text { on } R,
$$

the boundary conditions

$$
\begin{gathered}
v\left(0, x_{2}, x_{3}, t\right)=v_{D}\left(x_{2}, x_{3}, t\right), u\left(0, x_{2}, x_{3}, t\right)=u_{D}\left(x_{2}, x_{3}, t\right), \text { on }\{0\} \times D, \\
v\left(x_{1}, x_{2}, x_{3}, t\right)=u\left(x_{1}, x_{2}, x_{3}, t\right)=0, \text { on }(0, \infty) \times \partial D,
\end{gathered}
$$

and the asymptotic conditions

$$
u, v \rightarrow 0 \text {, uniformly as } x_{1} \rightarrow \infty \text {. }
$$

We assume that the nonlinear function $f(v)$ satisfies the condition

$$
-f(v) v \geq-\mu v^{2}, \quad \mu>0 .
$$


It is worth noting that the functions of the form $f(v)=-a|v|^{n} v+b|v|^{\frac{n}{2}} v+\mu_{1} v$, where $n, a$ and $\mu_{1}$ are positive constants satisfy condition (2.5) (see the Appendix for a proof).

The arguments of this section follow the ideas used to work for decay estimates for diffusion equations $[12,13,18]$. However, we need to use weight functions to save the mathematical difficulties proposed by several terms of the system. In our case, the analysis starts by considering the function

$$
F(z, t)=\int_{0}^{t} \int_{D(z)} \exp (-2 \omega s)\left(v^{2}+\delta u^{2}\right) d a d s
$$

where, from now on, $\omega$ is a positive constant to be selected later. Direct differentiation gives

$$
\frac{\partial F(z, t)}{\partial z}=2 \int_{0}^{t} \int_{D(z)} \exp (-2 \omega s)\left(v v_{, 1}+\delta u u_{, 1}\right) d a d s
$$

and

$$
\frac{\partial^{2} F(z, t)}{\partial z^{2}}=2 \int_{0}^{t} \int_{D(z)} \exp (-2 \omega s)\left[v_{, 1} v_{, 1}+v v_{, 11}+\delta\left(u_{, 1} u_{, 1}+u u_{, 11}\right)\right] d a d s .
$$

The use of the evolution equations, boundary conditions and initial conditions and the divergence theorem gives

$$
\begin{array}{r}
\frac{\partial^{2} F(z, t)}{\partial z^{2}}=2 \int_{0}^{t} \int_{D(z)} \exp (-2 \omega s)\left[v_{, i} v_{, i}+\delta u_{, i} u_{, i}-f(v) v+\omega v^{2}+(\gamma+\omega) u^{2}\right. \\
+(1-\sigma) u v] d a d s+\exp (-2 \omega t) \int_{D(z)}\left[v^{2}+u^{2}\right] d a .
\end{array}
$$

Poincaré's inequality for the functions defined on the domain $D$, the arithmetic-geometric inequality and condition (2.5) imply

$$
\begin{array}{r}
\frac{\partial^{2} F(z, t)}{\partial z^{2}} \geq 2 \int_{0}^{t} \int_{D(z)} \exp (-2 \omega s)\left[v_{, 1} v_{, 1}+\delta u_{, 1} u_{, 1}+\left(\omega+\lambda^{2}-\mu\right) v^{2}+\left(\omega-|\gamma|+\lambda^{2} \delta\right) u^{2}\right. \\
\left.-\frac{1}{2}|1-\sigma|\left(u^{2}+v^{2}\right)\right] d a d s+\exp (-2 \omega t) \int_{D(z)}\left[v^{2}+u^{2}\right] d a .
\end{array}
$$

Here, $\lambda^{2}$ is the first eigenvalue of the problem

$$
\Delta \varphi+\zeta \varphi=0 \text { on } D, \varphi=0 \text { on } \partial D .
$$

Then, we have

$$
\begin{aligned}
\frac{\partial^{2} F(z, t)}{\partial z^{2}} \geq 2 & \left(\omega+\lambda^{2}-\mu-\frac{1}{2}|1-\sigma|\right) \int_{0}^{t} \int_{D(z)} \exp (-2 \omega s) v^{2} d a d s \\
& +\frac{2}{\delta}\left(\omega-|\gamma|+\lambda^{2} \delta-\frac{1}{2}|1-\sigma|\right) \int_{0}^{t} \int_{D(z)} \exp (-2 \omega s) \delta u^{2} d a d s \\
& +2 \int_{0}^{t} \int_{D(z)} \exp (-2 \omega s) v_{, 1}^{2} d a d s+2 \int_{0}^{t} \int_{D(z)} \exp (-2 \omega s) \delta u_{, 1}^{2} d a d s \\
& +\int_{D(z)} \exp (-2 \omega t)\left[v^{2}+u^{2}\right] d a
\end{aligned}
$$


Now, we take $\omega$ sufficient large in order to guarantee that

$$
\omega+\lambda^{2}-\mu-\frac{1}{2}|1-\sigma| \text { and } \omega-|\gamma|+\lambda^{2} \delta-\frac{1}{2}|1-\sigma|
$$

are strictly positive.

Since $F \geq 0$ and after the use of the Schwarz inequality, we deduce that

$$
F \frac{\partial^{2} F(z, t)}{\partial z^{2}}-\frac{1}{2}\left(\frac{\partial F(z, t)}{\partial z}\right)^{2} \geq 2 M_{\omega} F^{2}+N F \frac{\partial F}{\partial t}
$$

where

$$
M_{\omega}=\min \left\{\omega+\lambda^{2}-\mu-\frac{1}{2}|1-\sigma|, \delta^{-1}\left(\omega-|\gamma|+\lambda^{2} \delta-\frac{1}{2}|1-\sigma|\right)\right\}, \quad N=\min \left\{1, \delta^{-1}\right\} .
$$

If we define the function

$$
J(z, t)=(F(z, t))^{1 / 2},
$$

inequality (2.13) implies that

$$
\frac{\partial^{2} J(z, t)}{\partial z^{2}} \geq M_{\omega} J+N \frac{\partial J}{\partial t} .
$$

If we consider the change of variable

$$
J(z, t)=\exp \left(-\frac{M_{\omega} t}{N}\right) \rho(z, t),
$$

the function $\rho$ satisfies the problem

$$
\begin{gathered}
\rho_{z z}-N \rho_{t} \geq 0, z>0, t>0, \\
\rho(z, 0)=0, z \geq 0, \\
\rho(0, t)=\exp \left(\frac{M_{\omega} t}{N}\right) P(t), t \geq 0, \\
\rho(z, t) \rightarrow 0 \text { (uniformly in } t \text { ) as } z \rightarrow \infty .
\end{gathered}
$$

Here, the function $P(t)$ is defined by

$$
P(t)=\left(\int_{0}^{t} \int_{D(0)} \exp (-2 \omega s)\left(v_{D}^{2}+\delta u_{D}^{2}\right) d a d s\right)^{1 / 2} .
$$

An upper bound for $\rho(z, t)$ in terms of the solution of an initial-boundary value problem for the one-dimensional heat equation now follows from the maximum principle. Let $y(z, t)$ be the solution of the problem

$$
\begin{gathered}
y_{z z}-N y_{t}=0, z>0, t>0, \\
y(z, 0)=0, z \geq 0, \\
y(0, t)=\exp \left(\frac{M_{\omega} t}{N}\right) P(t), t \geq 0,
\end{gathered}
$$




$$
y(z, t) \rightarrow 0 \text { (uniformly in } t \text { ) as } z \rightarrow \infty .
$$

The maximum principle yields

$$
\rho \leq y, z \geq 0, t \geq 0
$$

So we conclude

$$
J(z, t) \leq P(t) G(z, t)
$$

where

$$
\begin{aligned}
2 G(z, t)= & \exp \left(-\sqrt{M_{\omega}} z\right) \text { erfc }\left[\frac{N^{1 / 2} z}{2 t^{1 / 2}}-\left(\frac{M_{\omega} t}{N}\right)^{1 / 2}\right] \\
& +\exp \left(\sqrt{M_{\omega}} z\right) \operatorname{erfc}\left[\frac{N^{1 / 2} z}{2 t^{1 / 2}}+\left(\frac{M_{\omega} t}{N}\right)^{1 / 2}\right] .
\end{aligned}
$$

Here, $\operatorname{erfc}(x)$ is the complementary error function, which is defined by

$$
\operatorname{erfc}(x)=2(\pi)^{-1 / 2} \int_{x}^{\infty} \exp \left(-s^{2}\right) d s
$$

In view of the definition of $J$ and $F$, we obtain the estimate

$$
\left(\int_{0}^{t} \int_{D(z)}\left(v^{2}+\delta u^{2}\right) d a d s\right)^{1 / 2} \leq \exp (\omega t)\left(\int_{0}^{t} \int_{D(0)} \exp (-2 \omega s)\left(v_{D}^{2}+\delta u_{D}^{2}\right) d a d s\right)^{1 / 2} G(z, t)
$$

for $z \geq 0, t \geq 0$. Estimate (2.31) provides a mean-square estimate for the solutions of the problem (1.1), (1.3) with (2.1)-(2.5).

In order to obtain a sharper estimate for the decay, we recall the inequality (see [1], p. 298)

$$
\sqrt{\pi} \operatorname{erfc}(x)<\frac{1}{x} \exp \left(-x^{2}\right), x>0 .
$$

Therefore, we get that

$$
G(z, t) \leq \frac{2 N^{3 / 2} z(t / \pi)^{1 / 2} \exp \left(-\frac{M_{\omega} t}{N}\right)}{N^{2} z^{2}-4 M_{\omega} t^{2}} \exp \left(-\frac{N z^{2}}{4 t}\right)
$$

From (2.31) and (2.33) we obtain

$$
\left(\int_{0}^{t} \int_{D(z)}\left(v^{2}+\delta u^{2}\right) d a d s\right)^{1 / 2} \leq \exp (\omega t) P(t)\left[\frac{2 N^{3 / 2} z(t / \pi)^{1 / 2} \exp \left(-\frac{M_{\omega} t}{N}\right)}{N^{2} z^{2}-4 M_{\omega} t^{2}} \exp \left(-\frac{N z^{2}}{4 t}\right)\right]
$$

which is satisfied whenever $z>2 \sqrt{M_{\omega}} t / N$.

Inequality (2.34) shows that, for fixed $t$, the spatial decay is asymptotically controlled by the factor

$$
\exp \left(-\frac{N z^{2}}{4 t}\right)
$$

Thus, we have obtained the following theorem.

Theorem 2.1 Let $(u, v)$ be a solution of the problem determined by the system (1.1), (1.3), initial conditions (2.1) and boundary and asymptotic conditions (2.2)-(2.4). We further assume that the nonlinear function $f(v)$ satisfies (2.5). Then the spatial decay is asymptotically controlled by the factor given by (2.35). 


\section{Parabolic problem: $\delta=0$}

In this section we consider the parabolic problem of Section 2 in the limit case when $\delta=0$ under the extra condition $\sigma>0$. It corresponds to the coupling of a parabolic equation with an ordinary differential equation. First thing we can observe is that the arguments of Section 2 can be applied in this situation without difficulties. If we define $F$ as in (2.6) with $\delta=0$, we obtain

$$
\begin{aligned}
\frac{\partial^{2} F(z, t)}{\partial z^{2}}=2 \int_{0}^{t} \int_{D(z)} \exp (-2 \omega s)\left(v_{, i} v_{, i}-f(v) v+\omega v^{2}\right. & \left.+(\gamma+\omega) u^{2}+(1-\sigma) u v\right) d a d s \\
& +\int_{D(z)} \exp (-2 \omega s)\left[v^{2}+u^{2}\right] d a .
\end{aligned}
$$

We can adapt the arguments of Section 2 to obtain an inequality as (2.13), but in this case we can take $N=1$.

However, in this situation it is possible to prove a nonexistence result whenever we assume that the nonlinear function $f(v)$ satisfies a condition of the type

$$
-f(v) v \geq a|v|^{2 \alpha}-\mu v^{2}
$$

where $\alpha>1, \mu>0$ and $a>0$. Again the functions of the form $f(v)=-a|v|^{n} v+b|v|^{\frac{n}{2}} v+\mu_{1} v$, where $a, n$ and $\mu_{1}$ are positive constants satisfy our requirements (see the Appendix for a proof). Our arguments in this section are inspired by the papers [8, 9, 22]. Another time the use of weighted functions allows us to overcome the mathematical difficulties of the analysis.

If we consider the function

$$
\Phi(z, t)=-\int_{0}^{t} \int_{D(z)} \exp (-2 \omega s) v v_{, 1} d a d s,
$$

we get

$$
\begin{array}{r}
\frac{\partial \Phi(z, t)}{\partial z}=-\int_{0}^{t} \int_{D(z)} \exp (-2 \omega s)\left(v_{, i} v_{, i}-f(v) v+\omega v^{2}+\frac{\gamma+\omega}{\sigma} u^{2}\right) d a d s \\
-\frac{1}{2} \int_{D(z)} \exp (-2 \omega t)\left(v^{2}+\frac{1}{\sigma} u^{2}\right) d a
\end{array}
$$

It is worth noting that if we take $\omega \geq \max \{\mu,|\gamma|\}$ then

$$
-\frac{\partial \Phi(z, t)}{\partial z} \geq \int_{0}^{t} \int_{D(z)} \exp (-2 \omega s)\left(v_{, i} v_{, i}+a|v|^{2 \alpha}\right) d a d s .
$$

For arbitrary positive constant $\nu$, we have

$$
\begin{aligned}
& |\Phi(z, t)| \leq\left[\int_{0}^{t} \int_{D(z)} \exp (-2 \omega s) v^{2} d a d s \int_{0}^{t} \int_{D(z)} \exp (-2 \omega s) v_{, 1}^{2} d a d s\right]^{1 / 2} \\
& \leq M_{1}(t)\left[\left(\nu^{\alpha} \int_{0}^{t} \int_{D(z)} \exp (-2 \omega s)|v|^{2 \alpha} d a d s\right)^{\frac{1}{\alpha}}\left(\nu^{-1} \int_{0}^{t} \int_{D(z)} \exp (-2 \omega s) v_{, 1}^{2} d a d s\right)\right]^{1 / 2} \\
& \leq M_{1}(t)\left[\left(\nu^{\alpha} \int_{0}^{t} \int_{D(z)} \exp (-2 \omega s)|v|^{2 \alpha} d a d s\right)^{\frac{1}{\alpha+1}}\left(\nu^{-1} \int_{0}^{t} \int_{D(z)} \exp (-2 \omega s) v_{, 1}^{2} d a d s\right)^{\frac{\alpha}{\alpha+1}}\right]^{\frac{\alpha+1}{2 \alpha}},
\end{aligned}
$$


where

$$
M_{1}(t)=\left[\left(\int_{0}^{t} \exp (-2 \omega s) d s\right)^{\frac{\alpha-1}{\alpha}}|D|^{\frac{\alpha-1}{\alpha}}\right]^{1 / 2} .
$$

Using Young's inequality, we conclude that

$$
|\Phi(z, t)| \leq M_{2}(t)\left[\nu^{\alpha} \int_{0}^{t} \int_{D(z)} \exp (-2 \omega s)|v|^{2 \alpha} d a d s+\alpha \nu^{-1} \int_{0}^{t} \int_{D(z)} \exp (-2 \omega s) v_{, 1}^{2} d a d s\right]^{\frac{\alpha+1}{2 \alpha}}
$$

where

$$
M_{2}(t)=M_{1}(t) /(\alpha+1)^{(\alpha+1) / 2 \alpha} .
$$

Setting $\nu=(\alpha a)^{1 /(\alpha+1)}$, we obtain

$$
|\Phi(z, t)| \leq M_{3}(t)\left[-\frac{\partial \Phi(z, t)}{\partial z}\right]^{(\alpha+1) / 2 \alpha}
$$

where

$$
M_{3}(t)=M_{2}(t)\left[\alpha / a^{1 / \alpha}\right]^{1 / 2} .
$$

When we assume that $\Phi(0, t)<0$, we have that $\Phi(z, t)<0$ for all $z \geq 0$. Hence, we conclude that

$$
-\frac{\partial \Phi(z, t)}{\partial z} \geq\left[-\frac{\Phi(z, t)}{M_{3}(t)}\right]^{2 \alpha /(\alpha+1)}
$$

which, by integration, yields

$$
[-\Phi(z, t)]^{-(\alpha-1) /(1+\alpha)} \leq[-\Phi(0, t)]^{-(\alpha-1) /(1+\alpha)}-\frac{\alpha-1}{\alpha+1} M_{3}(t)^{-2 \alpha /(1+\alpha)} z .
$$

We note that the left hand side of (3.13) is positive, while for $z$ large enough, the right hand side is negative. So we have obtained the following result.

Theorem 3.1 Let $(u, v)$ be a solution of the problem determined by the system (1.1), (1.2) with initial conditions (2.1) and boundary conditions (2.2), (2.3). We further assume that the function $f(v)$ satisfies condition (3.2), where $\alpha>1, \mu>0$ and $a>0$. Therefore, no solution in the measure of $\Phi(z, t)$ can exist for all value of $z$, whenever $\Phi(0, t)<0$, where $\Phi(z, t)$ is defined by (3.3).

\section{Hyperbolic/parabolic problem}

In this section we study the asymptotic spatial behaviour of the solutions of the problem determined by the system (1.3)-(1.4) with conditions (2.1)-(2.4) and

$$
v_{t}(\boldsymbol{x}, 0)=0 \text {, on } R \text {. }
$$

It is worth noting that it corresponds to the coupling of a hyperbolic with a parabolic equation. Moreover, we do not impose the condition (2.5), but we assume some conditions that can be considered related. Thus, we suppose that

$$
-F(v)=-\int_{0}^{v} f(s) d s \geq-\mu^{*} v^{2},-f^{\prime}(v) \geq-\nu, \mu^{*}>0, \nu>0 .
$$


We will obtain a fast spatial decay estimate. Another time, the functions $f(v)=-a|v|^{n} v+$ $b|v|^{\frac{n}{2}} v+\mu_{1} v,\left(a, n\right.$ and $\mu_{1}$ are positive constants) satisfy our requirements (see the Appendix for a proof). In fact, we will prove that the decay is very similar to the one proposed for the parabolic problem. However, here we have a very different kind of coupling. The arguments of this section are inspired by the paper [20] and correspond to the use of the maximum principle argument proposed in the context of a coupling of parabolic and hyperbolic equations. It is worth noting that for the analysis proposed in this section (also the next one) the exponentially weighted Poincaré inequality plays a relevant role. When we analyze our problem, some terms of $u$ and $v$ in $L^{2}$-norm appear. We will control these terms with the help of inequality (1.5). That is, by means of the integral of the square of the time derivative. This will be an essential point in our analysis. It will be a fundamental ingredient to overcome the proposed mathematical difficulties. From now on we consider estimates for $0<t \leq t_{0}$, where $t_{0}$ is a fixed time.

In this situation the analysis starts by considering the function

$$
\Gamma(z, t)=-\int_{0}^{t} \int_{D(z)} \exp (-2 \omega s)\left(\delta u_{, 1} u+v_{, 1} v_{, s}\right) d a d s
$$

If we use the divergence theorem, the evolution equations, the boundary conditions, the asymptotic behaviour and the initial conditions, we have

$$
\begin{aligned}
\Gamma(z, t)= & \int_{0}^{t} \int_{z}^{\infty} \int_{D(\eta)} \exp (-2 \omega s)\left[u u_{, s}+\delta u_{, i} u_{, i}-\sigma v u+\gamma u^{2}\right. \\
& \left.+\epsilon v_{, s} v_{, s s}+\left(1-\epsilon f^{\prime}(v)\right) v_{, s}^{2}+v_{, i} v_{, i s}-f(v) v_{, s}+u v_{, s}\right] d V d s \\
& =\frac{1}{2} \int_{R(z)} \exp (-2 \omega t)\left(u^{2}+\epsilon v_{, t}^{2}+v_{, i} v_{, i}-2 F(v)\right) d V \\
& +\int_{0}^{t} \int_{R(z)} \exp (-2 \omega s)\left[\omega\left(u^{2}+\epsilon v_{, s}^{2}+v_{, i} v_{, i}-2 F(v)\right)+\delta u_{, i} u_{, i}-\sigma v u\right. \\
& \left.+\gamma u^{2}+\left(1-\epsilon f^{\prime}(v)\right) v_{, s}^{2}+u v_{, s}\right] d V d s .
\end{aligned}
$$

In view of the conditions (4.2), we can see that

$$
\begin{aligned}
\Gamma(z, t) \geq & \frac{1}{2} \int_{R(z)} \exp (-2 \omega t)\left(u^{2}+\epsilon v_{, t}^{2}+v_{, i} v_{, i}-2 \mu^{*} v^{2}\right) d V \\
& +\int_{0}^{t} \int_{R(z)} \exp (-2 \omega s)\left[\omega\left(u^{2}+\epsilon v_{, s}^{2}+v_{, i} v_{, i}-2 \mu^{*} v^{2}\right)\right. \\
& \left.\quad+\delta u_{, i} u_{, i}-\sigma v u+\gamma u^{2}+\left(1-\epsilon f^{\prime}(v)\right) v_{, s}^{2}+u v_{, s}\right] d V d s .
\end{aligned}
$$

In order to control some summands of $\Gamma(z, t)$ we will consider the following estimates. We 
have

$$
\begin{aligned}
& \frac{1}{2} \int_{R(z)} \exp (-2 \omega s)\left(-2 \mu^{*} v^{2}\right) d V=-\mu^{*} \int_{0}^{t} \int_{R(z)} \frac{d}{d s}\left[\exp (-2 \omega s) v^{2}\right] d V d s \\
& =2 \omega \mu^{*} \int_{0}^{t} \int_{R(z)} \exp (-2 \omega s) v^{2} d V d s-2 \mu^{*} \int_{0}^{t} \int_{R(z)} \exp (-2 \omega s) v v_{, s} d V d s \\
& \geq 2 \omega \mu^{*} \int_{0}^{t} \int_{R(z)} \exp (-2 \omega s) v^{2} d V d s \\
& \quad-2 \mu^{*}\left(\int_{0}^{t} \int_{R(z)} \exp (-2 \omega s) v^{2} d V d s\right)^{1 / 2}\left(\int_{0}^{t} \int_{R(z)} \exp (-2 \omega s) v_{, s}^{2} d V d s\right)^{1 / 2}
\end{aligned}
$$

From the exponentially weighted Poincaré inequality, we get

$$
\begin{aligned}
& \frac{1}{2} \int_{R(z)} \exp (-2 \omega s)\left(-2 \mu^{*} v^{2}\right) d V \geq 2 \omega \mu^{*} \int_{0}^{t} \int_{R(z)} \exp (-2 \omega s) v^{2} d V d s \\
& -2 \mu^{*}\left(\frac{4 t^{2}}{\pi^{2}+4 t^{2} \omega^{2}}\right)^{1 / 2}\left(\int_{0}^{t} \int_{R(z)} \exp (-2 \omega s) v_{, s}^{2} d V d s\right)^{1 / 2}\left(\int_{0}^{t} \int_{R(z)} \exp (-2 \omega s) v_{, s}^{2} d V d s\right)^{1 / 2} \\
& =2 \omega \mu^{*} \int_{0}^{t} \int_{R(z)} \exp (-2 \omega s) v^{2} d V d s-\frac{4 \mu^{*} t_{0}}{\left(\pi^{2}+4 t_{0}^{2} \omega^{2}\right)^{1 / 2}} \int_{0}^{t} \int_{R(z)} \exp (-2 \omega s) v_{, s}^{2} d V d s .
\end{aligned}
$$

On the other hand,

$$
\begin{aligned}
& -\sigma \int_{0}^{t} \int_{R(z)} \exp (-2 \omega s) u v d V d s \\
& \geq-\frac{\sigma}{2} \int_{0}^{t} \int_{R(z)} \exp (-2 \omega s) u^{2} d V d s-\frac{\sigma}{2} \frac{4 t_{0}^{2}}{\pi^{2}+4 t_{0}^{2} \omega^{2}} \int_{0}^{t} \int_{R(z)} \exp (-2 \omega s) v_{, s}^{2} d V d s
\end{aligned}
$$

Moreover,

$\int_{0}^{t} \int_{R(z)} \exp (-2 \omega s) u v_{, s} d V d s \geq-\frac{1}{2} \int_{0}^{t} \int_{R(z)} \exp (-2 \omega s) u^{2} d V d s-\frac{1}{2} \int_{0}^{t} \int_{R(z)} \exp (-2 \omega s) v_{, s}^{2} d V d s$.

We can select $\omega$ so large to guarantee that

$$
\left[\omega+\gamma-\frac{\sigma+1}{2}\right] u^{2}+\left[\omega \epsilon-\frac{1}{2}-\frac{4 \mu^{*} t_{0}}{\left(\pi^{2}+4 t_{0}^{2} \omega^{2}\right)^{1 / 2}}-\frac{2 \sigma t_{0}^{2}}{\pi^{2}+4 t_{0}^{2} \omega^{2}}+\left(1-\epsilon f^{\prime}(v)\right)\right] v_{, s}^{2} \geq \varepsilon^{*}\left(u^{2}+v_{, s}^{2}\right),
$$

for some $\varepsilon^{*}>0$, for all $u, v_{, t}$ and so $\Gamma(z, t) \geq 0$, for all $u, v, v_{, t}, v_{, i}, u_{, i}$. In view of the estimates we see that

$$
\Gamma(z, t) \geq C \int_{0}^{t} \int_{R(z)}\left(u^{2}+v_{s}^{2}+u_{, i} u_{, i}+v^{2}+v_{, i} v_{, i}\right) d V d s
$$

for some $C>0$. 
Now, we define the function which is assumed to exist

$$
E(z, t)=\int_{z}^{\infty} \Gamma(\xi, t) d \xi
$$

To guarantee the existence of the function $E(z, t)$, from the definition of $\Gamma(z, t)$ by $(4.3)$, it is sufficient to assume that $u, u_{, 1}, v_{, 1}, v_{, s} \rightarrow 0$ as $x^{-1}$ uniformly on $t, x_{2}, x_{3}$. We have

$$
\frac{\partial E(z, t)}{\partial z}=-\Gamma(z, t)
$$

and

$$
\begin{aligned}
& \frac{\partial^{2} E(z, t)}{\partial z^{2}}=\frac{1}{2} \int_{D(z)} \exp (-2 \omega t)\left(u^{2}+\epsilon v_{, t}^{2}+v_{, i} v_{, i}-2 F(v)\right) d a \\
& +\int_{0}^{t} \int_{D(z)} \exp (-2 \omega s)\left[\omega\left(u^{2}+\epsilon v_{, s}^{2}+v_{, i} v_{, i}-2 F(v)\right)+\delta u_{, i} u_{, i}-\sigma v u\right. \\
& \left.+\gamma u^{2}+\left(1-\epsilon f^{\prime}(v)\right) v_{, s}^{2}+u v_{, s}\right] d a d s .
\end{aligned}
$$

From the definition of $E$ and $\Gamma$, we also have that

$$
\frac{\partial E(z, t)}{\partial t}=-\exp (-2 \omega t) \int_{R(z)}\left(\delta u_{, 1} u+v_{, 1} v_{, t}\right) d V=\exp (-2 \omega t)\left(-\int_{R(z)} v_{, 1} v_{, t} d V+\frac{\delta}{2} \int_{D(z)} u^{2} d a\right) .
$$

From the A-G inequality, we can obtain a positive constant $\beta$, which can depend on time $t_{0}$, such that

$$
\left|\exp (-2 \omega t) \int_{R(z)} v_{, 1} v_{, t} d V\right| \leq \frac{1}{2} \exp \left(2 \omega t_{0}\right) \int_{R(z)}\left(v_{, 1}^{2}+v_{, t}^{2}\right) d V \leq-\beta \frac{\partial E}{\partial z} .
$$

We note that previous estimates (4.7)-(4.10) are also valid on $D(z)$ instead of $R(z)$. In fact, we can obtain them by a similar analysis in $D(z)$. However, we do not include it here. Therefore we get

$$
\exp (-2 \omega t) \frac{\delta}{2} \int_{D(z)} u^{2} d a \leq \delta \frac{\partial^{2} E}{\partial z^{2}}
$$

Estimates (4.16) and (4.17) imply that

$$
\frac{\partial E(z, t)}{\partial t} \leq-\beta \frac{\partial E}{\partial z}+\delta \frac{\partial^{2} E}{\partial z^{2}} .
$$

A direct consequence of the previous estimates (see [20]) is that

$$
E(z, t) \leq \exp (K z-Q t) \sup _{0 \leq s \leq t}[\exp (Q s) E(0, s)] \frac{z}{(4 \pi \delta)^{1 / 2}} \int_{0}^{t}(t-s)^{-3 / 2} \exp \left(-\frac{z^{2}}{4 \delta(t-s)}\right) d s
$$

where

$$
K=\frac{\beta}{2 \delta}, Q=K^{2} \delta
$$


Let us denote

$$
M(z, t)=\frac{z}{(4 \pi \delta)^{1 / 2}} \int_{0}^{t} s^{-3 / 2} \exp \left(-\frac{z^{2}}{4 \delta s}\right) d s
$$

Then, we obtain

$$
E(z, t) \leq \exp (K z-Q t) \sup _{0 \leq s \leq t}[\exp (Q s) E(0, s)] M(z, t) .
$$

If we make the change of variable $\tau^{2}=z^{2} / 4 \delta s$, we see that

$$
M(z, t)=\operatorname{erfc} \frac{z}{(4 \delta t)^{1 / 2}} .
$$

If we recall the estimate (2.32), we get

$$
E(z, t) \leq \frac{A(t)}{z} \exp \left(K z-\frac{z^{2}}{4 \delta t}\right)
$$

where

$$
A(t)=(4 \delta t)^{1 / 2} \exp (-Q t) \sup _{0 \leq s \leq t}[\exp (Q s) E(0, s)] .
$$

Estimate (4.24) is very similar to the one obtained in the study of the parabolic version of the system. The main difference is the multiplication by the function $\exp (K z)$. But for large values of $z$ the behaviour is very similar. We have proved the following theorem.

Theorem 4.1 Let $(u, v)$ be a solution of the problem determined by the system (1.3), (1.4) with conditions (2.1)-(2.4) and (4.1). We further assume that the function $f(v)$ satisfies (4.2). Then the spatial decay is asymptotically controlled by the factor $\exp \left(K z-\frac{z^{2}}{4 \delta t}\right)$.

\section{Hyperbolic problem: $\delta=0$}

In this section we study the problem of Section 4 in the particular case $\delta=0$. That is, we consider the system (1.2), (1.4) with conditions (2.1)-(2.4) and (4.1). We do not impose the condition (2.5), but the conditions (4.2). Our system corresponds to a couple of a hyperbolic equation with an ordinary differential equation. The arguments of this section are inspired by the papers $[3,4,7]$. Again the exponentially weighted Poincaré inequality plays a relevant role to overcome the new difficulties proposed by the system. In fact the use of this inequality allows us to recover classical arguments to this new situation. If we define the function

$$
\Gamma(z, t)=-\int_{0}^{t} \int_{D(z)} \exp (-2 \omega s) v_{, 1} v_{, s} d a d s
$$

we obtain

$$
\begin{aligned}
& \Gamma(z+h, t)-\Gamma(z, t)=-\frac{1}{2} \int_{z}^{z+h} \int_{D(z)} \exp (-2 \omega t)\left(u^{2}+\epsilon v_{, t}^{2}+v_{, i} v_{, i}-2 F(v)\right) d V \\
& -\int_{0}^{t} \int_{z}^{z+h} \int_{D(z)} \exp (-2 \omega s)\left(\omega\left(u^{2}+\epsilon v_{, s}^{2}+v_{, i} v_{, i}-2 F(v)\right)-\sigma v u+\gamma u^{2}+\left(1-\epsilon f^{\prime}(v)\right) v_{, s}^{2}+u v_{, s}\right) d V d s
\end{aligned}
$$


Hence,

$$
\begin{aligned}
& \frac{\partial \Gamma(z, t)}{\partial z}=-\frac{1}{2} \int_{D(z)} \exp (-2 \omega t)\left(u^{2}+\epsilon v_{, t}^{2}+v_{, i} v_{, i}-2 F(v)\right) d a \\
& -\int_{0}^{t} \int_{D(z)} \exp (-2 \omega s)\left(\omega\left(u^{2}+\epsilon v_{, s}^{2}+v_{, i} v_{, i}-2 F(v)\right)-\sigma v u+\gamma u^{2}+\left(1-\epsilon f^{\prime}(v)\right) v_{, s}^{2}+u v_{, s}\right) d a d s .
\end{aligned}
$$

From (5.1) we find that

$$
\frac{\partial \Gamma(z, t)}{\partial t}=-\exp (-2 \omega t) \int_{D(z)} v_{, 1} v_{, t} d a .
$$

It is clear that we can obtain a similar analysis in $D(z)$ to the one proposed in the previous section for $R(z)$. However, we do not include it here to write a shorter paper. In a similar way to (4.16), we can obtain the following inequality

$$
\left|\frac{\partial \Gamma(z, t)}{\partial t}\right|+\tau \frac{\partial \Gamma(z, t)}{\partial z} \leq 0,
$$

where $\tau$ is a calculable positive constant which also depends on time $t_{0}$. From (5.5) we get

$$
\frac{\partial \Gamma(z, t)}{\partial t}+\tau \frac{\partial \Gamma(z, t)}{\partial z} \leq 0
$$

and

$$
\frac{\partial \Gamma(z, t)}{\partial t}-\tau \frac{\partial \Gamma(z, t)}{\partial z} \geq 0
$$

Integrating (5.6) we obtain

$$
\Gamma\left(z, \tau^{-1}\left(z-z^{*}\right)\right) \leq 0, \quad z \geq z^{*} .
$$

Similarly, integrating (5.7) it follows

$$
\Gamma\left(z, \tau^{-1}\left(z^{* *}-z\right)\right) \geq 0, \quad z^{* *} \geq z .
$$

Inequalities (5.8) and (5.9) imply that, for each finite time $t$, the asymptotic condition

$$
\lim _{z \rightarrow \infty} \Gamma(z, t)=0
$$

holds.

Now we consider

$$
\begin{aligned}
& \mathcal{E}_{\omega}(z, t)=\frac{1}{2} \int_{z}^{\infty} \int_{D(\eta)} \exp (-2 \omega t)\left(u^{2}+\epsilon v_{, t}^{2}+v_{, i} v_{, i}-2 F(v)\right) d V \\
& +\int_{0}^{t} \int_{z}^{\infty} \int_{D(\eta)} \exp (-2 \omega s)\left(\omega\left(u^{2}+\epsilon v_{, s}^{2}+v_{, i} v_{, i}-2 F(v)\right)-\sigma v u+\gamma u^{2}\right. \\
& \left.\quad+\left(1-\epsilon f^{\prime}(v)\right) v_{, s}^{2}+u v_{, s}\right) d V d s .
\end{aligned}
$$

Therefore, (5.6) implies that

$$
\mathcal{E}_{\omega}(z, t) \leq \mathcal{E}_{\omega}\left(z^{*}, 0\right)
$$


where $z, z^{*}$ and $t$ are related by $t=\tau^{-1}\left(z-z^{*}\right)$. In a similar way, we get

$$
\mathcal{E}_{\omega}(z, t) \geq \mathcal{E}_{\omega}\left(z^{* *}, 0\right)
$$

for $t=\tau^{-1}\left(z^{* *}-z\right)$. From (5.12) and (5.13) we conclude that

$$
\mathcal{E}_{\omega}(z, t) \leq \mathcal{E}_{\omega}\left(z^{*}, t^{*}\right)
$$

for $\left|t-t^{*}\right| \leq \tau^{-1}\left(z-z^{*}\right)$. Hence, we have proved the following result.

Theorem 5.1 Let $(u, v)$ be a solution of the initial-boundary-value problem (1.2), (1.4) satisfying (2.1)-(2.4) and (4.1)-(4.2). Then, the energy function $\mathcal{E}_{\omega}(z, t)$ defined in (5.11) satisfies inequality (5.14) whenever $\left|t-t^{*}\right| \leq \tau^{-1}\left(z-z^{*}\right)$, where $\tau$ is a positive constant satisfying (5.5).

If we define the measure

$$
\mathcal{E}_{\omega}^{*}(z, t)=\int_{0}^{t} \mathcal{E}_{\omega}(z, s) d s
$$

the following inequalities can be obtained as in [3]:

$$
\begin{gathered}
\mathcal{E}_{\omega}^{*}(z, t) \leq 0, \quad \tau t \leq z \\
\mathcal{E}_{\omega}^{*}(z, t) \leq \exp (2 \omega t)\left(1-\frac{z}{\tau t}\right) \mathcal{E}_{\omega}^{*}(0, t), \quad \tau t \geq z .
\end{gathered}
$$

It is worth noting that (5.16) implies that the solutions of our problem vanish whenever $\tau t \leq z$. That is $u=v=0$, for $\tau t \leq z$. This is a domain of influence type result. Hence, we have proved:

Corollary 5.1 Let $(u, v)$ be a solution of the system (1.2), (1.4) satisfying (2.1)-(2.4) and (4.1)-(4.2). Then $u=v=0$, when $\tau t \leq z$.

\section{Further comments and conclusions}

The arguments proposed in the above sections also can be applied to the one-dimensional case. We mean the case that $R$ corresponds to a semi-infinite line. Therefore, the condition (2.3) has no meaning and the condition $(2.2)$ becomes $v(0, t)=v_{D}(t), u(0, t)=u_{D}(t)$.

In this paper we have proved the following results:

(1) For the parabolic problem determined by (1.1), (1.3), (2.1)-(2.5), the solutions satisfy the decay $(2.35)$, for fixed $t$. That is the decay is asymptotically controlled by $\exp \left(-\frac{N z^{2}}{4 t}\right)$, where $N$ is given by $(2.14)$.

(2) For the parabolic problem determined by (1.1), (1.2) with $\sigma>0$ and conditions (2.1)(2.4) and (3.2), we obtain a nonexistence result. That is, no solution in the measure of $\Phi(z, t)$ can exist for all value of $z$, whenever $\Phi(0, t)<0$, where $\Phi(z, t)$ is defined by $(3.3)$.

(3) For the hyperbolic/parabolic problem determined by (1.3)-(1.4), (2.1)-(2.4), satisfying (4.1) and (4.2), the spatial decay of solutions is asymptotically controlled by the factor $\exp \left(K z-\frac{z^{2}}{4 \delta t}\right)$, where $K$ is given in $(4.20)$. 
(4) For the hyperbolic problem determined by (1.2)-(1.4) with (2.1)-(2.4), satisfying (4.1)(4.2), the energy function $\mathcal{E}_{\omega}(z, t)$ defined by (5.11) satisfies $\mathcal{E}_{\omega}(z, t) \leq \mathcal{E}_{\omega}\left(z^{*}, t^{*}\right)$, whenever $\left|t-t^{*}\right| \leq \tau^{-1}\left(z-z^{*}\right)$, where $\tau$ is a positive constant satisfying (5.5). Moreover, the solutions of our problem vanish, i.e., $u=v=0$, whenever $\tau t \leq z$. This is a "domain of influence" result.

Acknowledgments The authors thank to the anonymous referee for his/her useful comments. This work is part of the project "Análisis Matemático de las Ecuaciones en Derivadas Parciales de la Termomecánica" (MTM2013-42004-P) submitted to the Spanish Ministry of Economy and Competitiveness.

\section{References}

[1] M. Abramowitz and I.A. Stegun (eds.), Handbook of Mathematical Functions, Dover Publications, New York, 1965.

[2] G.N. Aliyeva and V.K. Kalantarov, Structural stability for Fitzhugh-Nagumo Equations, Appl. Comput. Math. 10 (2011) 289-293.

[3] F. Bofill and R. Quintanilla, Spatial estimates for dynamical problems in several elasticity theories, Ricerche di Matematica 46 (1997) 425-441.

[4] S. Chirita and R. Quintanilla, Saint-Venant's principle in linear elastodynamics, J. Elasticity 42 (1996) 201-215.

[5] R. FitzHugh, Impulses and physiological states in theoretical models of nerve membrane, Biophys. J. 1 (1961) 445-466.

[6] J.N. Flavin, R.J. Knops and L.E. Payne, Decay estimates for the constrained elastic cylinder of variable cross-section, Quarterly Applied Mathematics 47 (1989) 325-350.

[7] J.N. Flavin, R.J. Knops and L.E. Payne, Energy bounds in dynamical problems for a semiinfinite elastic beam, in: Elasticity: Mathematical Methods and Applications (Eason, G., Ogden, R. W. eds.), Chichester: Ellis Horwood, 1989, pp. 101-111.

[8] J.N. Flavin, R.J. Knops and L.E. Payne, Asymptotic behaviour of solutions to semi-linear elliptic equations on the half-cylinder, J. Appl. Math. Phys. (ZAMP) 43 (1992) 405-417.

[9] J.N. Flavin, R.J. Knops, and L.E. Payne, Asymptotic and other estimates for a semilinear elliptic equation in a cylinder, Quarterly Jour. Mech. Appl. Math. 45 (1992) 617-627.

[10] W. Gao and J. Wang, Existence of wavefronts and impulses to FitzHughNagumo equations, Nonlinear Analysis: Theory, Methods \& Applications 57 (2004) 667-676.

[11] A.L. Hodgkin and A.F. Huxley, A quantitative description of membrane current and its application to conduction and excitation in nerve, J. Physiol. 117 (1952) 500-544.

[12] C.O. Horgan and R. Quintanilla, Spatial decay of transient end effects in functionally graded heat conducting materials, Quarterly of Applied Mathematics 59 (2001) 529-542. 
[13] C.O. Horgan, L.E. Payne and L.T. Wheeler, Spatial decay estimates in transient heat conduction, Quarterly of Applied Mathematics 42 (1984) 119-127.

[14] C.-H. Hsu, T.-H. Yang and C.-R. Yang, Diversity of traveling wave solutions in FitzHughNagumo type equations, Journal of Differential Equations 247 (2009) 1185-1205.

[15] M.C. Leseduarte and R. Quintanilla, Phragmén-Lindelöf alternative for an exact heat conduction equation with delay, Communications on Pure and Applied Analysis 12 (2013) 1221-1235.

[16] H.M. Lieberstein, On the Hodgkin-Huxley partial differential equation, Math. Biosci. 1 (1967) 45-69.

[17] Y. Lv and W. Wang, Limit dynamics for the stochastic FitzHughNagumo system, Nonlinear Analysis: Real World Applications 11 (2010) 3091-3105.

[18] A. Miranville and R. Quintanilla, Spatial decay for several phase-field models, ZAMM. 93 (2013) 801-810.

[19] J. Nagumo, S. Arimoto and S. Yoshizawa, An active pulse transmission line simulating nerve axon, Proc. IRE. 50 (1962) 2061-2070.

[20] R. Quintanilla, Damping of end effects in a thermoelastic theory, Applied Mathematics Letters 14 (2001) 137-141.

[21] R. Quintanilla, Spatial asymptotic behaviour in incremental thermoelasticity, Asymptotic Analysis 27 (2001) 265-279.

[22] R. Quintanilla, On the spatial blow-up and decay for some nonlinear parabolic equations with nonlinear boundary conditions, J. Appl. Math. Phys. (ZAMP) 57 (2006) 595-603.

[23] A. Slavova, and P. Zecca, CNN model for studying dynamics and travelling wave solutions of FitzHughNagumo equation, Journal of Computational and Applied Mathematics 151 (2003) 13-24.

[24] J.C. Song, Phragmén-Lindelöf and continuous dependence type results in a Stokes flow, Appl. Math. Mech. 31 (2010) 875-882.

[25] J.C. Song, Asymptotic and other estimates for semilinear parabolic problem in semi-infinite cylinder, Appl. Math. Mech. 32 (2011) 1333-1338.

[26] M. València, On invariant regions and asymptotic bounds for semilinear partial differential equations, Nonlinear Analysis: Theory, Methods \& Applications 14 (1990) 217-230.

[27] M. València, Invariant regions and asymptotic bounds for a hyperbolic version of the nerve equation, Nonlinear Analysis: Theory, Methods \& Applications 16 (1991) 1035-1052. 


\section{Appendix}

Here, we shall prove some properties of the function $f(v)$ appearing in the systems of equations for the different sections.

(A) The functions $f(v)=-a|v|^{n} v+b|v|^{\frac{n}{2}} v+\mu_{1} v$, where $a, \mu_{1}, n>0$ satisfy the condition $(2.5)$.

In fact, we have that

$$
f(v) v=-a|v|^{n+2}+b|v|^{\frac{n}{2}} v^{2}+\mu_{1} v^{2}=-a|v|^{n+2}+b|v|^{\frac{n}{2}+1}|v|+\mu_{1} v^{2} .
$$

From the A-G inequality, for $\varepsilon>0$, it follows

$$
f(v) v \leq-a|v|^{n+2}+\frac{b \varepsilon}{2}|v|^{n+2}+\frac{b}{2 \varepsilon} v^{2}+\mu_{1} v^{2} \leq\left(-a+\frac{|b| \varepsilon}{2}\right)|v|^{n+2}+\frac{|b|}{2 \varepsilon} v^{2}+\mu_{1} v^{2} .
$$

We can take $\varepsilon>0$ such that $-a+\frac{|b| \varepsilon}{2}=0$, that is $\varepsilon=\frac{2 a}{|b|}>0$. Therefore,

$$
f(v) v \leq \frac{b^{2}}{4 a} v^{2}+\mu_{1} v^{2} \leq \mu v^{2}
$$

for $\mu \geq \frac{b^{2}}{4 a}+\mu_{1}$, and the result follows.

(B) The functions $f(v)=-a|v|^{n} v+b|v|^{\frac{n}{2}} v+\mu_{1} v$, where $a, \mu_{1}, n>0$ satisfy the condition (3.2).

Now we prove the claim. We consider $\alpha=1+\frac{n}{2}>1$. Then,

$$
f(v) v=-a|v|^{n+2}+b|v|^{\frac{n}{2}+1}|v|+\mu_{1} v^{2} .
$$

Again, from the A-G inequality, it follows

$$
f(v) v \leq\left(-a+\frac{|b| \varepsilon}{2}\right)|v|^{n+2}+\frac{|b|}{2 \varepsilon} v^{2}+\mu_{1} v^{2} .
$$

We can take $\varepsilon>0$ such that $-a+\frac{|b| \varepsilon}{2}=\frac{a}{2}$, that is $\varepsilon=\frac{a}{|b|}>0$. Hence,

$$
f(v) v \leq \frac{-a}{2}|v|^{n+2}+\frac{b^{2}}{2 a} v^{2}+\mu_{1} v^{2} \leq \frac{-a}{2}|v|^{2 \alpha}+\left(\frac{b^{2}}{2 a}+\mu_{1}\right) v^{2} \leq \mu v^{2},
$$

for $\mu \geq \frac{b^{2}}{2 a}+\mu_{1}$.

(C) The functions $f(v)=-a|v|^{n} v+b|v|^{\frac{n}{2}} v+\mu_{1} v$, where $a, \mu_{1}, n>0$ satisfy (4.2).

We prove the first inequality.

$$
\begin{aligned}
F(v)=\int_{0}^{v} f(s) d s & =\int_{0}^{v}\left[-a|s|^{n} s+b|s|^{\frac{n}{2}} s+\mu_{1} s\right] d s \\
& =v^{2}\left[\frac{-a}{n+2}|v|^{n}+\frac{2 b}{n+4}|v|^{n / 2}+\frac{\mu_{1}}{2}\right] .
\end{aligned}
$$


Since $-a /(n+2)<0$, there exist two constants $M_{1}<M_{2}$ such that

$$
\frac{-a}{n+2}|v|^{n}+\frac{2 b}{n+4}|v|^{n / 2}+\frac{\mu_{1}}{2}<0
$$

if $v<M_{1}$ or $v>M_{2}$. Let us consider $>0, \mu^{*} \geq \max _{v \in\left[M_{1}, M_{2}\right]}\left[\frac{-a}{n+2}|v|^{n}+\frac{2 b}{n+4}|v|^{n / 2}+\frac{\mu_{1}}{2}\right]$. So, $F(v) \leq \mu^{*} v^{2}$ and the result follows.

Now, we prove the second inequality. An easily computation gives us that

$$
f^{\prime}(v)=-a(n+1)|v|^{n}+b\left(\frac{n}{2}+1\right)|v|^{n / 2}+\mu_{1} .
$$

Since $-a(n+1)<0$, there exist two constants $K_{1}<K_{2}$ such that $f^{\prime}(v)<0$ whenever $v<K_{1}$ or $v>K_{2}$. Let us consider $\nu>0, \nu \geq \max _{v \in\left[K_{1}, K_{2}\right]}\left[f^{\prime}(v)\right]$. Therefore, $-f^{\prime}(v) \geq-\nu$. 